\title{
РАЗРАБОТКА И ПРИМЕНЕНИЕ МЕТОДОВ МАШИННОГО ОБУЧЕНИЯ И АЛГОРИТМОВ РЕШЕНИЯ ЗАДАЧ УПРАВЛЕНИЯ И ПРИНЯТИЯ РЕШЕНИЙ В ХОЗЯЙСТВЕННОЙ ДЕЯТЕЛЬНОСТИ АГРОПРОМЫШЛЕННОГО ПРЕДПРИЯТИЯ
}

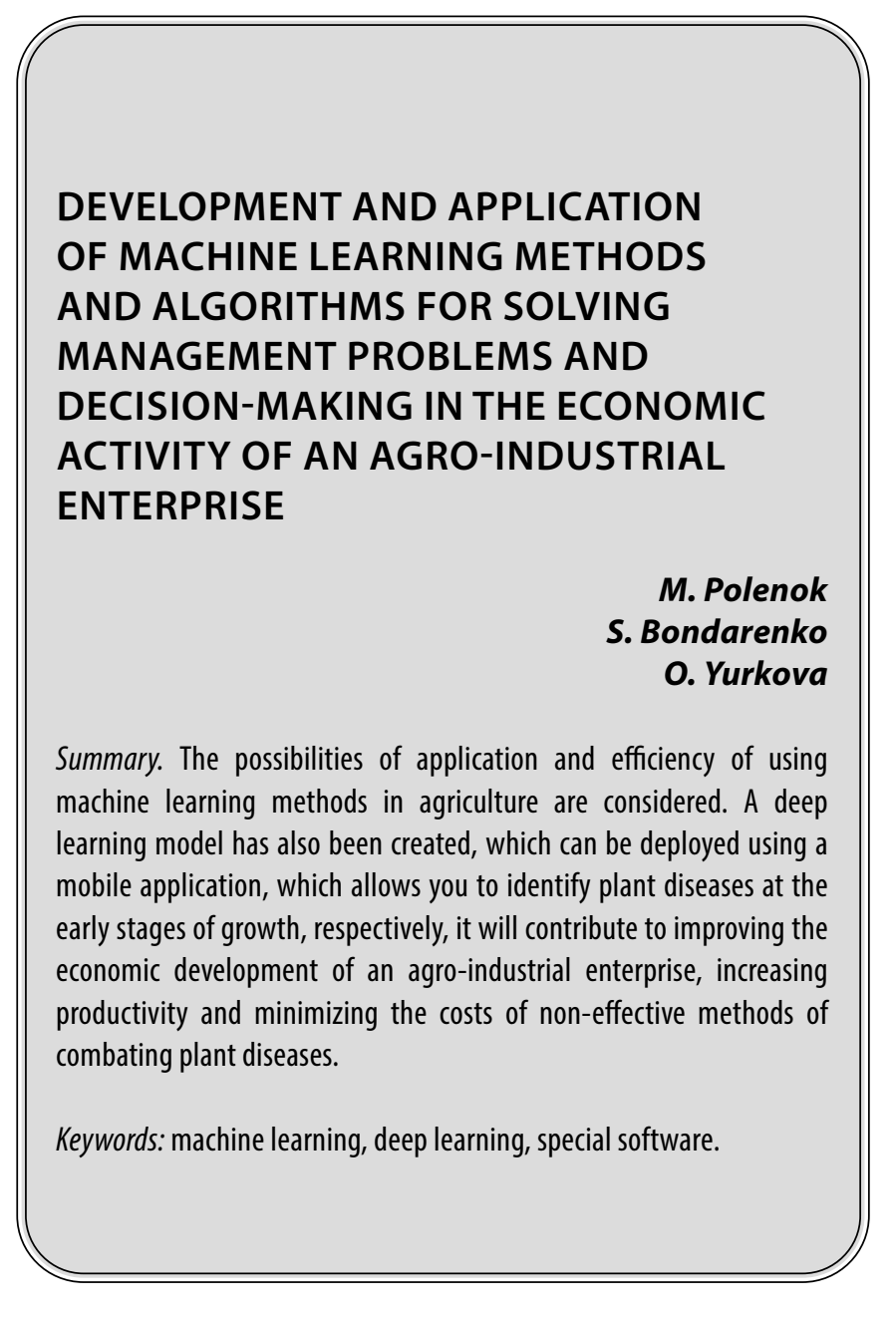

\section{Введение}

егодня технологии искусственного интеллекта применяются в различных сферах человеческой деятельности, в том числе и в сельском хозяйстве.

Целью нашего исследования является рассмотрение возможностей применения и эффективного использования методов машинного обучения в сельском хозяйстве. Технологии искусственного интеллекта, применяемые в сельском хозяйстве, обладают определенной спецификой. Искусственный интеллект выполняет такие функции как распознавание образов, осущест-

\author{
Поленок Максим Викторович \\ ФГБОУ ВО «Брянский государственный инженерно- \\ технологический университет» \\ polenok.maksim.2001@mail.ru \\ Бондаренко Сергей Владимирович \\ ФГБОУ ВО «Брянский государственный инженерно- \\ технологический университет» \\ Bondrenkoseregabondarenko576@gmail.com \\ Юркова Ольга Николаевна \\ К.э.н., ФГБОУ ВО «Брянский государственный \\ инженерно-технологический университет» \\ yurkova_olga@mail.ru
}

Аннотация. Рассмотрены возможности применения и эффективность использования методов машинного обучения в сельском хозяйстве. Так же создана модель глубокого обучения, которую можно развернуть с помощью мобильного приложения, что позволяет определить болезни растений на ранних стадиях роста, соответственно, будет способствовать улучшению экономического развития агропромышленного предприятия, повышая урожайность и минимизирую затраты на не эффективные методы борьбы с болезнями растений.

Ключевые слова: машинное обучение, глубокое обучение, специальное программное обеспечение. вление самообучения. Благодаря внедрению искусственного интеллекта в отраслях сельского хозяйства повышается производительность труда, повышается эффективность управленческих решений, что приводит к расширению возможностей человека на рабочем месте.

Теоретические аспекты машинного обучения в Аеятельности агропромышленных преАприятий

Не так давно получил распространение термин «большие данные», обозначающий новую прикладную 


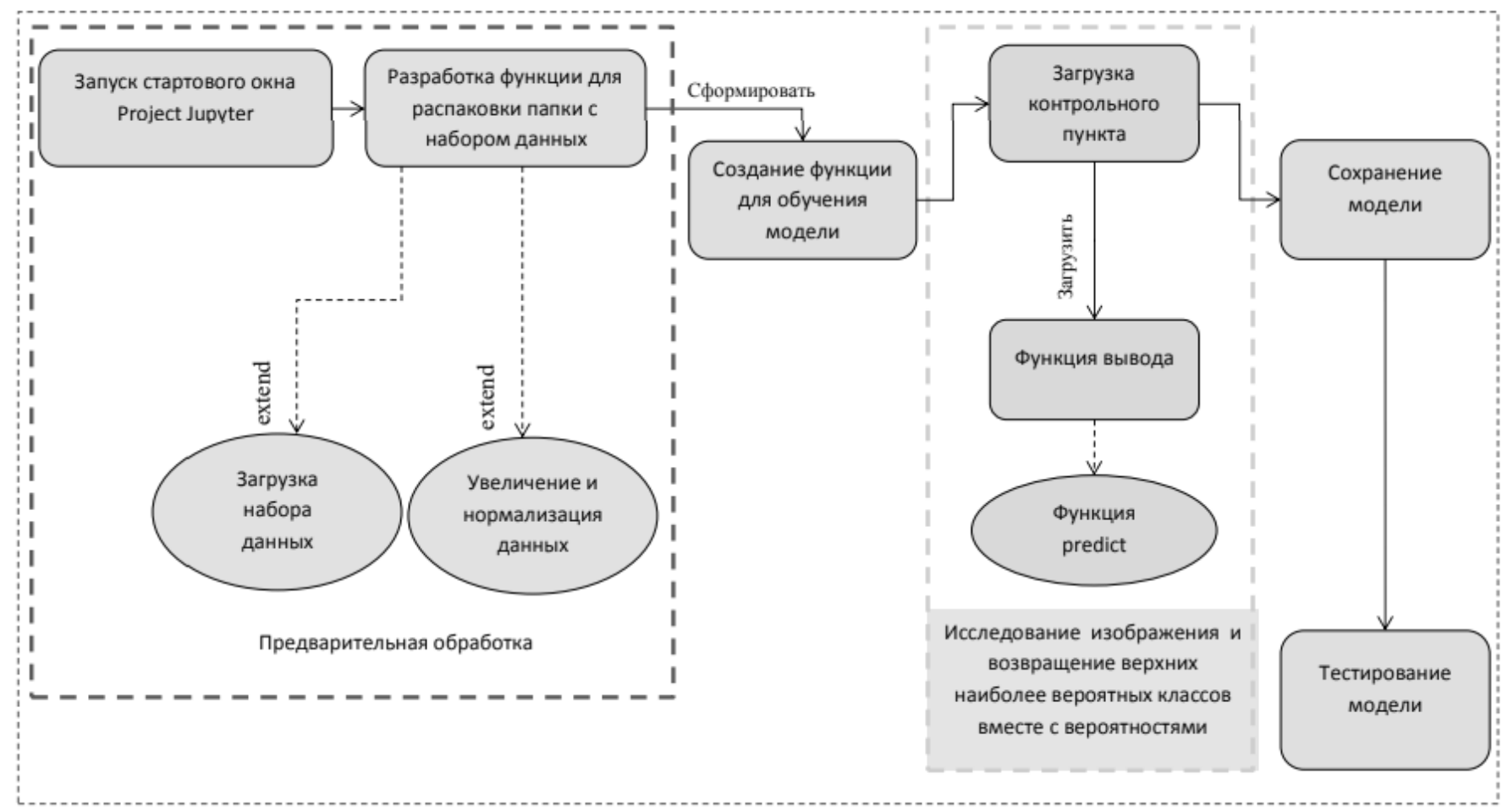

Рис. 1. Разработка функциональной модели для выявления болезней сельскохозяйственных культур по изображению с использованием системы Deep Learning

область - поиск методов автоматического быстрого анализа огромных объемов данных.

Наука о больших данных может успешно решать множество различных задач: прогнозирование зарплаты по вакансии, оценка состояния печени по кардиограмме, прогнозирование числа заболеваний у пациентов в следующем месяце. Самым перспективным подходом к анализу больших данных принято считать применение машинного обучения - набор функций, используя которые ЭВМ способен находить в массивах данных взаимосвязи и закономерности.

Машинное обучение возникло из-за стремления к созданию и использованию искусственного интеллекта. Оно использует данные для определения того, какой алгоритм является лучшим для получения результатов.

В наше время данные слишком велики для того, чтобы обрабатывать их вручную, но машинное обучения прекрасно справляется с этой задачей. Для этого оно использует спектр на основе метода оптимизации большого количества параметров.

Одной из областей применения технологий машинного обучения становится отрасль сельского хозяйства. Для того, чтобы прокормить весь мир в будущем, нам необходимо улучшить методы ведения сельского хозяйства. Применение искусственного интеллекта может сыграть ключевую роль в решении этого вопроca.

В основе новой эры ведения сельского хозяйства лежит концепция, использующая технологии для наблюдения за культурами, измерения различных показателей и реагирования на их изменения.

В настоящее время технологии машинного обучения уже во всю внедряются в сельское хозяйство. Методы машинного обучения позволяют обрабатывать огромное количество входных данных о развитии растений и на этой основе производить точное прогнозирование урожайности культур. Одними из примеров его использования является: обнаружение заболеваний растений, классификация и идентификация сорняков.

Выделяют этапы внедрения машинного обучения в процессы сельского хозяйства:

- Постановка задач;

- Сбор, хранение и предобработка данных;

- Создание и обучение алгоритма;

- Разработка высокоуровневой обвязки;

- Интеграция;

- Сбор обратной связи, корректировка модели. 


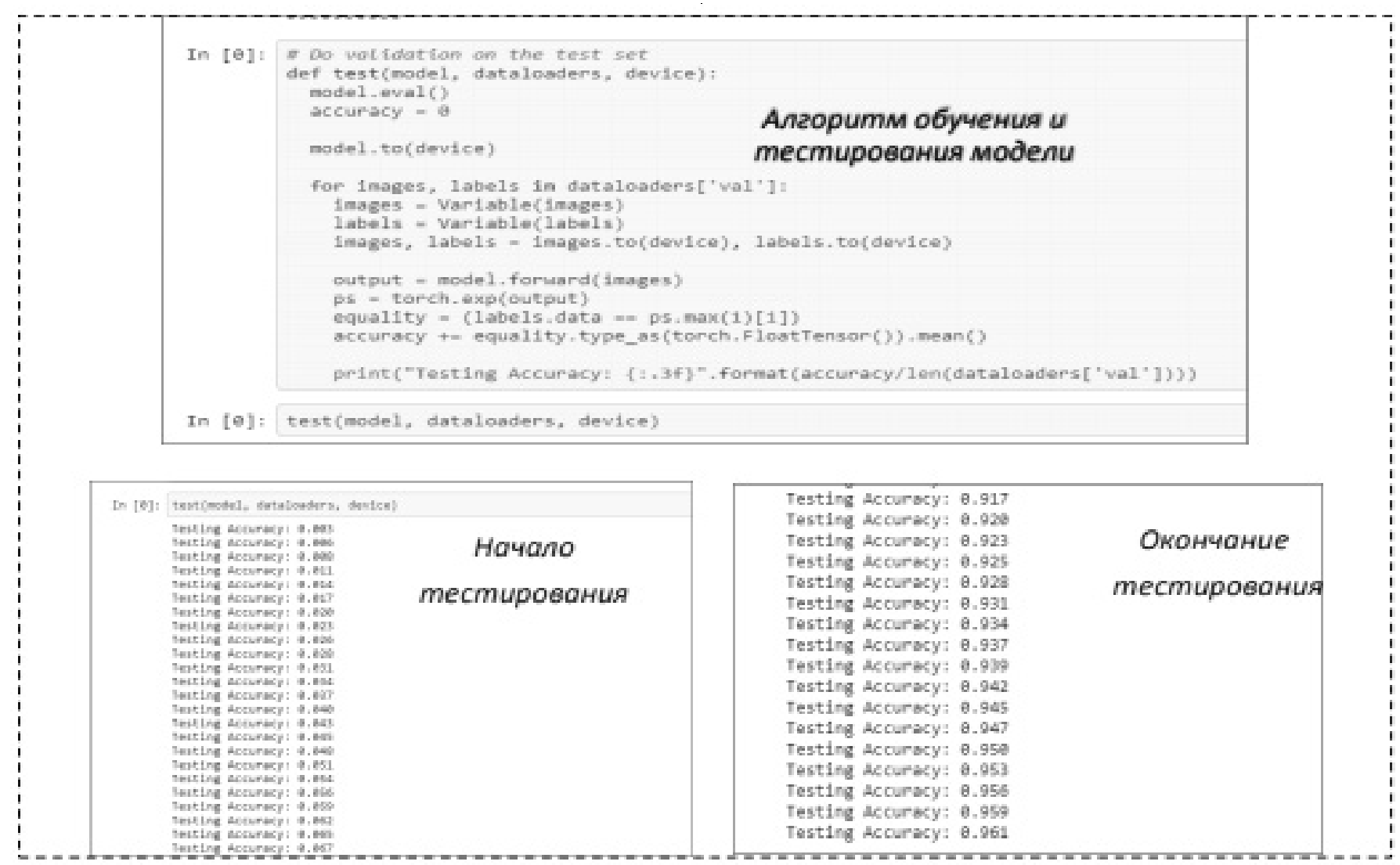

Рис. 2. Обучение и тестирование модели

\section{Разработка функபиональной моАели $А$ выявления болезней сельскохозяЙственных КУльтУР по изображению с использованием системы Deep Learning}

Перед тем, как использовать алгоритмы машинного обучения, необходимо ответственно подойти к выбору правильных признаков, на основе которых будет производиться обучение. Наиболее важным он является для распознавания по изображению. Обычно выбор набора признаков для обучения во многом определяет успешность работы алгоритма. Так, для задачи распознавания изображений в качестве таких признаков может служить преобладающий цвет в изображении, степень его изменения, наличие на изображении четких границ.

Для реализации подхода deep learning в современной науке используются многослойные нейронные сети различных архитектур. На рисунке 1 представлена разработка функциональной модели для выявления болезней сельскохозяйственных культур по изображению с использованием системы Deеp Learning.
Для разработки функциональной модели были проанализированы способы выявления болезней растений по изображениям листьев с использованием системы глубокого обучения. Первым этапом необходимо загрузить файл с набором исходных изображений для дальнейшего обучения.

Для выполнения данной работы был выбран набор данных, представленный по адресу: https://github.com/ spMohanty/PlantVillageDataset/ tree/master/raw/color. Пакет Torchvision состоит из популярных наборов данных, архитектур моделей и шаблонов для компьютерного зрения. Набор данных разбивается на две части: обучение и проверка.

Перед началом работы необходимо загрузить набор данных, а также исходных данных для классификации, сопоставляющий целочисленные кодированные категории с реальными названиями болезней растений. Ниже представлен код этих действий.

\# Загрузите набор данных и распакуйте папку

gdown https://drive.google.com/ucid=1Bhh3VeMBH6 F7vKqHdDDmdyi7RfvQNqJ 
tar-xvf PlantVillage.tar.gz

import json

with open('categories.json', 'r') as f:

cat_to_name $=$ json.load(f)

Далее необходимо представить функцию, необходимую для обучения модели:

\section{\#Функция для обучения модели}

def train_model(model, criterion, optimizer, scheduler, num_epochs=20):

since $=$ time .time()

best_model_wts = copy.deepcopy $($ model.state_dict ()$)$

best_acc $=0.0$

for epoch in range(1, num_epochs+1):

print('Epoch \{\}$/\{\}$ '.format(epoch, num_epochs))

print $\left({ }^{\prime}-{ }^{*} * 10\right)$

time_elapsed $=$ time.time ()$-$ since

print('Training complete in $\{: .0 f\} m$ $\{: .0 f\} s$ '.format(

time_elapsed // 60, time_elapsed\% 60))

print('Best valid accuracy: $\{: 4 f\}$ '.format(best_acc))

Следующим этапом является этап обучения и тестирования модели. Ознакомиться с кодом алгоритма обучения и тестирования модели можно на рисунке 2.

На следующем этапе необходимо написать функцию, принимающую изображение и модель. Функция на основе полученных данных должна предсказывать класс болезни растения на изображении. Ниже представлен код такой функции:

def predict(image_path, model, topk $=5$ ):

image $=$ torch.FloatTensor ([process_image(Image. open(image_path))]) model.eval() output $=$ model. forward(Variable(image))

probabilities=torch.exp(output).data.numpy()[0]

top_idx=np.argsort(pobabilities)[-topk:][::-1]

top_class=[idx_to_class[x] for $\mathrm{x}$ in top_idx]

top_probability=pobabilities[top_idx]

return top_probability, top_class

\section{Реализация функциональной мохели системы с помошью препроцессинга изобрахений}

Препроцессинг изображений - это предварительная обработка изображений. Основной целью предобработки, в нашем случае, является выявление наиболее важной части изображения и удалении ненужного шума.

Изображение RGB можно просматривать как три изображения (изображение с красной шкалой, изобра- жение с зеленой шкалой и изображение с синей шкалой), накладываемые друг на друга.

Для начала необходимо исследовать методы, которые будут использоваться для предварительной обработки и извлечения объектов. Чтобы произвести тестирования и обработку мы будем использовать в качестве примера одно изображение листа растения.

Для предварительной обработки изображения были выполнены следующие шаги:

- Преобразование RGB в изображение в градациях серого.

- Сглаживание изображения с использованием фильтра Гасса.

- Адаптивный порог изображения с помощью метода порога Оцу.

- Закрытие дыр с помощью морфологического преобразования.

- Извлечение границ с использованием контуров.

Для начала необходимо подключить все необходимые библиотеки и прочитать тестовое изображение. Код данных действий представлен ниже.

import os import cv2

import numpy as np

from matplotlib import pyplot as plt

\%matplotlib inline

ds_path = "Flavia leaves dataset" In [3]:

test_img_path $=$ ds_path + “\\2546.jpg” test_img_path

Следующим этапом является преобразование RGB-изображения в изображение в градации серого, а также необходимо произвести сглаживание изображения с использованием фильтра Гаусса. Будем использовать сглаживание изображения с помощью гауссовского фильтра размером $(25,25)$. В результате изображение становится более размытым, что позволит нашей модели лучше его считывать.

Преимущества размытия:

- Это помогает в удалении шума.

- Ребра низкой интенсивности удаляются.

- Это помогает скрыть детали при необходимости.

Код данных преобразований представлен ниже.

gs = cv2.cvtColor(img, cv2.COLOR_RGB2GRAY)

blur $=$ cv2.GaussianBlur(gs, $(25,25), 0)$ 
Далее нам необходимо получить черно-белое изображение, для этого будем использовать адаптивную пороговую обработку изображений с использованием метода пороговой обработки Otsu.

Алгоритм позволяет разделить пиксели двух классов: полезные и фоновые.

ret_otsu, im_bw_otsu $=$ cv2.threshold $($ blur, $0,255, \mathrm{cv} 2$. THRESH_BINARY_INV+CV2.THRESH_OTSU)

В результате, изображение получилось черно-белым, но на нем видны своеобразные отверстия, которые будут мешать корректному считыванию изображений. Поэтому необходимо произвести закрытие отверстий.

Основные методы преобразования:

- Erode - размывание (операция сужения)

- Dilate - растягивание (операция расширения)

И последним пунктом предварительно обработки является функция извлечения границ с использованием контуров. Контуры используются для извлечения границ листьев.

contours, hierarchy $=\mathrm{cv} 2$.findContours(closing, cv2. RETR_TREE, cV2.CHAIN_APPROX_SIMPLE)

plottedContour $=\mathrm{cv} 2$.drawContours(gs, contours, $-1,(0,255,0), 10)$
Таким образом, в ходе разработки функциональной модели системы с помощью исследования методов предварительной обработки и извлечения объектов мы преобразовали модель для более точного распознавания.

\section{Зак^ючение}

В заключение хочется сказать, что сегодня технологии искусственного интеллекта уже используются во многих сферах и отраслях человеческой деятельности. В сельском хозяйстве также используется искусственный интеллект, осуществляющий такие функции как обнаружение болезней растений, классификация растений, подсчет плодов, классификация земельных ресурсов, прогнозирование погоды, изучение поведения животных.

Итогом работы служит достижение цели, т.к. были рассмотрены возможности применения и эффективность использования методов машинного обучения в сельском хозяйстве. Так же создана модель глубокого обучения. Это приложение определяет болезни растений на ранних стадиях роста, что будет способствовать улучшению экономического развития агропромышленного предприятия, повышая урожайность и минимизирую затраты на не эффективные методы борьбы с болезнями растений.

\section{ЛИТЕРАТУРА}

1. Геловани В. А., Башлыков А.А., Бритков В.Б., Вязилов Е.Д. Интеллектуальные системы поддержки принятия решений в нештатных ситуациях с использованием информации о состоянии природной среды; Едиториал УРСС - М., 2015. - 304 c.

2. Гудвин Г.К., Гребе С.Ф., Сальгадо М.Э. Проектирование систем управления (+ CD-ROM); Бином. Лаборатория знаний — M., 2016. - 912 c.

3. Мерков А.Б. Распознавание образов. Введение в методы статистического обучения; Едиториал УРСС — М., 2016. — 256 с.

4. Искусственный интеллект и принятие решений, № 1, 2011; Ленанд — М., 2019. — 742 с.

5. Искусственный интеллект и принятие решений, № 4, 2011: моногр.; ИСА РАН — М., 2019. - 124 с.

6. Мартин Дж. Организация баз данных в вычислительных системах; Мир — М., 2016. — 664 с.

7. Флах Петер Машинное обучение. Наука и искусство построения алгоритмов, которые извлекают знания из данных. Учебник; ДМК Пресс - М., 2015. - $400 \mathrm{c}$. 